\title{
Si and SiGe Nanowires: Fabrication Process and Thermal Conductivity Measurement by $3 \omega$-Scanning Thermal Microscopy
}

\author{
Stéphane Grauby, ${ }^{* \dagger}{ }^{\dagger}$ Etienne Puyoo, ${ }^{\dagger, \ddagger, \S}$ Jean-Michel Rampnoux, ${ }^{\dagger}$ Emmanuelle Rouvière, ${ }^{\ddagger}$ \\ and Stefan Dilhaire ${ }^{\dagger}$ \\ ${ }^{\dagger}$ University Bordeaux, LOMA, CNRS UMR 5798, F 33400 Talence, France \\ ${ }^{\ddagger}$ CEA DRT/LITEN/DTNM, LCRE, 17 Rue des Martyrs, 38054 Grenoble cedex, France \\ ${ }^{\S}$ INSA de Lyon, INL, CNRS UMR 5270, 7 Avenue Jean Capelle, 69621 Villeurbanne Cedex, France
}

\begin{abstract}
We have grown various samples of Si and SiGe nanowires (NWs), either by a classical vapor-liquid-solid (VLS) process or by chemical etching, to measure their thermal conductivity and thus evaluate their efficiency for thermoelectrics applications. To do so, we have chosen a $3 \omega$ Scanning Thermal Microscopy (SThM) imaging technique which is until now the only method able to perform topographical and thermal measurements simultaneously on an assembly of individual NWs, leading to a statistical value of their thermal conductivity. A size effect is clearly observed on Si NWs: $50 \mathrm{~nm}$ diameter NWs offer a reduced thermal conductivity in comparison with $200 \mathrm{~nm}$ diameter or even larger NWs. On the contrary, the thermal conductivity of SiGe NWs is widely reduced in comparison with the SiGe bulk value, even for large diameters, bigger than Si NWs ones. We discuss our results, comparing them with thermal conductivity values from the literature obtained by other measurement methods or models.
\end{abstract}

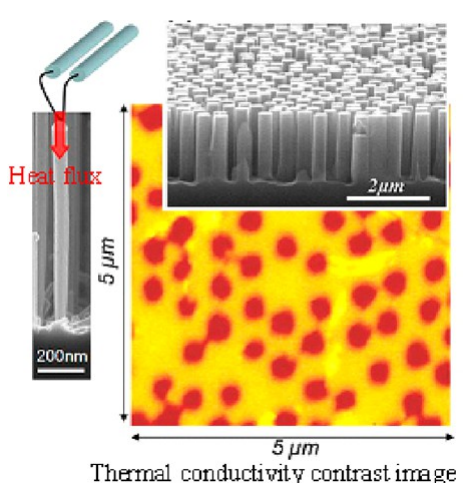

\section{INTRODUCTION}

Thermoelectricity is a field of great interest, and the efficiency of a thermoelectric material is determined by its figure of merit $Z T=\left(\left(S^{2} \sigma\right) /(\lambda)\right) T$ where $S, \sigma, \lambda$, and $T$ are, respectively, the Seebeck coefficient, the electrical conductivity, the thermal conductivity, and the temperature. During the years, the most commonly used thermoelectric material was bulk $\mathrm{Bi}_{2} \mathrm{Te}_{3}{ }_{3}{ }^{1}$ and its room temperature figure of merit was limited to 1 at maximum. Over the past decade, the development of nanostructures $^{2-6}$ has attracted attention because of their potential capacity to reduce the thermal conductivity without reducing the electrical conductivity and therefore to behave as a phonon glass and an electron crystal. ${ }^{7,8}$ This can be the case in nanostructured materials for which at least one dimension is smaller than the phonon mean free path and larger than the electron mean free path. As a consequence, one dimensional systems such as nanowires (NWs) can be very good candidates for thermoelectric applications. There is hence a real double challenge: the first one is the development of a chemical process able to control the composition, size, spacing, and surface state of the NWs, and the second one is the measurement of the thermal conductivity of nanometric structures.

Until now, Si NWs were the most commonly studied NWs. Indeed, silicon $(\mathrm{Si})$ is very abundant and the most used semiconductor in microelectronics, and its processing is current and low cost. In addition, $\mathrm{Si} \mathrm{NWs}$ seem to be very promising $^{8-10}$ since numerous theoretical studies have shown that when the diameter of a $\mathrm{Si}$ wire becomes smaller than the phonon mean free path, i.e., $300 \mathrm{~nm}$ at room temperature, its thermal conductivity can be reduced in comparison with bulk $\mathrm{Si}^{11-13}$ This thermal conductivity reduction has also been observed experimentally. However, the experimental inves tigations on this topic do not abound in the literature. A few thermal conductivity measurements of individual Si NWs have been realized with microfabricated suspended devices. ${ }^{9,14,15}$ This technique is powerful because the Seebeck coefficient and the electrical conductivity ${ }^{8,9,15}$ are evaluated at the same time. These two other physical properties are indeed of great interest for thermoelectrics. Moreover, the measurements with this type of microfabricated devices can be performed over a wide temperature range, which is helpful to understand the underlying physics. However, it is currently the only technique available to measure the thermal conductivity of individual one dimensional (1D) nanostructures, which explains why there are not many experimental data in the literature. Nevertheless, it is worth mentioning that this method, which measures only one $\mathrm{NW}$ at the same time, requires many heavy processing steps and leads to the oxidation of the surface of the NWs since they are not embedded in a matrix but in contact with air. In addition, this technique measures the thermal conductivity of one NW isolated from its matrix which can be quite different from the thermal conductivity of the NWs embedded in the matrix, which constitutes the effective functioning device, because of the matrix/NW interaction. ${ }^{16}$ We have proposed an experımentai proceaure ${ }^{17}$ basea on Jcannıng Inermai 


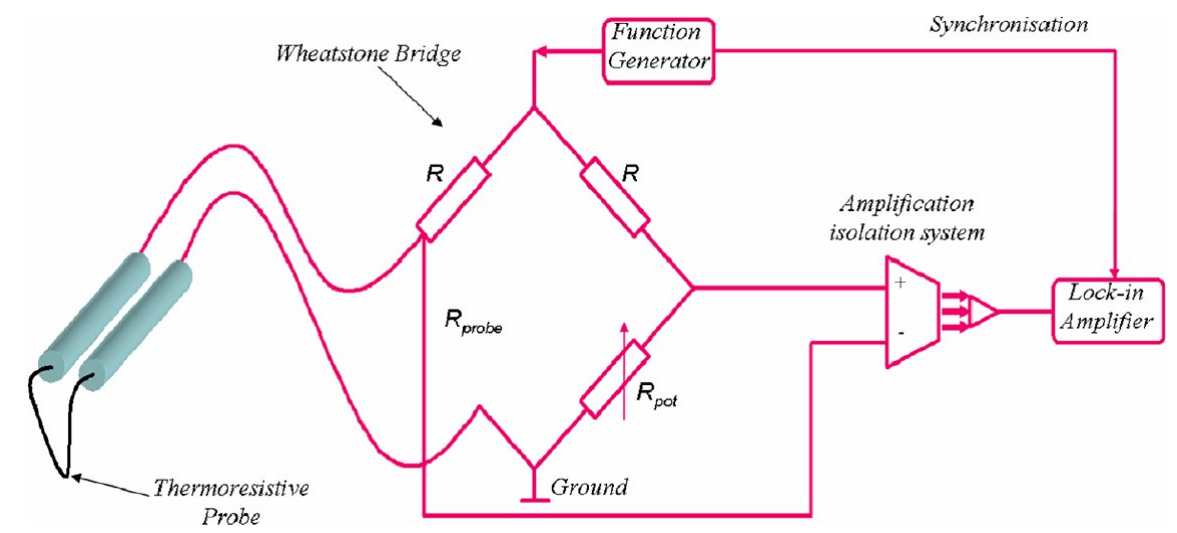

Figure 1. $3 \omega$ scanning thermal microscopy principle.

Microscopy (SThM $)^{18,19}$ which allows us to carry out thermal images of individual NWs with a $100 \mathrm{~nm}$ typical thermal spatial resolution and a $10 \mathrm{~nm}$ typical topographical spatial resolution. This technique has the advantage to thermally probe a wide range of individual NWs embedded in their matrix in a few minutes. We can hence deduce a mean thermal conductivity of the NWs by a statistical data processing.

In this paper, we first recall, in Section 2, the principle of the measurement setup as well as the experimental conditions. Then, we present results obtained on NWs obtained by two different growing processes. The first investigated NWs, presented in Section 3, are $50 \mathrm{~nm}$ diameter $\mathrm{Si}$ NWs grown using a classical vapor-liquid-solid (VLS) process. A thermal conductivity range could be identified for these NWs. Nevertheless, we underline the difficulty to perform thermal measurements on such small NWs and to obtain a precise value of their thermal conductivity. Then, other $\mathrm{Si}$ NWs with diameters varying from 200 to $400 \mathrm{~nm}$, hence easier to experimentally characterize, have been synthesized (Section 4.1) by an original metal assisted chemical wet etching process that enables us to precisely control the size and spacing of the NWs. Their thermal conductivity is estimated in Section 4.2, and we show the interest of having a wide diameter dispersion on the same set of samples. Silicon germanium nanowires ( $\mathrm{SiGe} \mathrm{NWs}$ ) were also fabricated by the same chemical wet etching process, and we show in Section 4.3 that they might be even more promising than $\mathrm{Si}$ NWs for thermoelectric applications. In all cases, the NWs are surrounded by a $\mathrm{SiO}_{2}$ matrix on their whole length. Finally, in the last section, we discuss the interest of the various kinds of investigated NWs and the validity of our results, comparing them with available data from the literature.

\section{EXPERIMENTAL METHODOLOGY}

The measurements are performed by using SThM (Scanning Thermal Microscopy) in a $3 \omega$ method configuration ${ }^{20-22}$ at room temperature. The most commonly used probe is the well known thermoresistive Wollaston tip ${ }^{22,23}$ made of a $5 \mu \mathrm{m}$ diameter platinum rhodium core covered with a $250 \mu \mathrm{m}$ diameter silver coating which has been suppressed at the tip apex over a $200 \mu \mathrm{m}$ length. This tip reveals to offer a limited spatial resolution-hardly submicrometric - and a low cutoff frequency ( $\mathrm{a}$ few hundred $\mathrm{Hz}$ ), limiting the acquisition speed. ${ }^{24}$ To overcome these limitations, the tip used here is a new commercial $\mathrm{Pd} / \mathrm{SiO}_{2}$ thermoresistive probe to carry out thermal images while simultaneously obtaining contact mode topography images. It is made of a thin palladium (Pd) ribbon on a $1 \mu \mathrm{m}$ thick $\mathrm{SiO}_{2}$ silica layer. The thin Pd ribbon acts as the thermoresistive element. Its temperature coefficient has been measured: $\alpha_{\text {Tip }}=1.38 \times 10^{-3} \mathrm{~K}^{-1}$. The probe electrical resistance is $R_{0}=368 \Omega$. The length and width of the Pd tip are measured from Scanning Electron Microscopy (SEM) images and also estimated by an analytic thermal model describing the probe out of contact to 8.8 and $2.6 \mu \mathrm{m}$, respectively. ${ }^{19}$ Its thermal cutoff frequency is typically $3 \mathrm{kHz}$ and its spatial resolution $100 \mathrm{~nm},{ }^{24}$ but these performances can slightly vary from one tip to another.

The probe is included in a Wheatstone bridge connected to an amplification system (Figure 1). The variable resistor $R_{\text {pot }}$ is adjusted so that its electrical resistance should be equal to the probe electrical resistance. A $\omega$ pulsation sinusoidal current $I(t)$ passes through the thermoresistive probe. A heat flux $P_{\text {Joule }}$ at pulsation $2 \omega$ is dissipated in the probe generating a temperature variation $T_{2 \omega}$ at the same pulsation. The thermoresistive probe resistance is then modulated at $2 \omega$. Finally, according to Ohm's law, the tip voltage is modulated at $3 \omega$, and its amplitude can be expressed as ${ }^{17}$

$$
\left(V_{3 \omega}\right)_{\text {Tip }}=K_{\text {ampli }} \frac{R_{\text {Tip }} \alpha_{\text {Tip }} I_{0}}{2}\left\langle T_{2 \omega}\right\rangle
$$

where $K_{\text {ampli }}$ is the amplification system gain, $I_{0}$ the amplitude of the $\omega$ pulsation current supplying the probe, and $\left\langle T_{2 \omega}\right\rangle$ the mean temperature variation amplitude over the tip length $L$.

Experimentally, a lock in measurement of the $3 \omega$ tip voltage $\left(V_{3 \omega}\right)_{\text {Tip }}$ is made during each scan in contact with a sample. We can then deduce a $\left\langle T_{2 \omega}\right\rangle$ tip temperature variations map and then an equivalent tip-sample thermal resistance $R_{\text {eq. }}$. Indeed, when the tip comes into contact with a material, a heat flow goes from the tip to the sample, and this flow depends on the sample thermal conductance. Consequently, the $\left\langle T_{2 \omega}\right\rangle$ tip temperature variations depend on the equivalent thermal resistance $R_{\text {eq }}$ between the tip and the sample. The more conductive the sample, the lower the $2 \omega$ thermal variations. This configuration is hence sometimes called conductivity contrast imaging.

Let us consider a NW on top of which the tip is positioned to do a $3 \omega \mathrm{SThM}$ measurement (Figure 2). The equivalent thermal resistance $R_{\text {eq }}$ can be modeled by four thermal resistances in series: the tip to sample contact thermal resistance $R_{\mathrm{C}}$, the constriction resistance $R_{\text {Tip-NW }}$ of the heat flux between the tip and the NW, the sample thermal intrinsic resistance $R_{\mathrm{NW}}=\left(1 / \lambda_{\mathrm{NW}}\right)(L / S)$, and the constriction resistance 


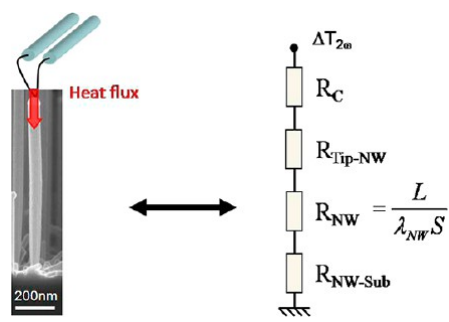

Figure 2. Equivalent thermal schema of the thermal flux passing from the tip to a NW.

$R_{\mathrm{NW} \text {-Sub }}$ of the heat flux between the NW and the substrate. The thermal conductivity of the NW is then given by

$$
\lambda_{\mathrm{NW}}=\frac{L}{S} \times \frac{1}{R_{\mathrm{eq}}-R_{\mathrm{c}}-R_{\mathrm{Tip}-\mathrm{NW}}-R_{\mathrm{NW}-\mathrm{Sub}}}
$$

where $L$ and $S$ are, respectively, the NW length and section.

Let us underline that, for a sample constituted of several NWs, this experimental method enables us to simultaneously measure $R_{\mathrm{eq}}$ on each NW of the thermal image. Then it enables us to do a statistical data processing, hence to deduce a mean thermal conductivity and the associated standard deviation, provided that you are able to determine the values of the three other thermal resistances $R_{\mathrm{C}}, R_{\mathrm{Tip}-\mathrm{NW}}$, and $R_{\mathrm{NW}-\mathrm{Sub}}$, which depend on the tip and sample studied but also on the atmospheric conditions. Then, a specific procedure is necessary for each sample. Let us note that the experimental setup enables us to work from atmospheric pressure to a $10^{-5}$ Torr vacuum.

\section{THERMAL CONDUCTIVITY OF VLS SILICON NANOWIRES}

The first kind of sample is an assembly of Si NWs grown by chemical vapor deposition and embedded in a silica $\mathrm{SiO}_{2}$ die. $\mathrm{Si}$ NWs have been grown via Au catalyzed Vapor-Liquid-Solid (VLS) reaction in an epitaxial chamber ${ }^{25-27}$ under a $\mathrm{SiH}_{4}$ vapor flow. NW growth is typically performed at $650{ }^{\circ} \mathrm{C}$ with a $\mathrm{SiH}_{4}$ flow rate of $20 \mathrm{sccm}$ leading to a 10 Torr partial pressure. $\mathrm{N}$ type doping is eventually carried out by simultaneously injecting gaseous $\mathrm{PH}_{3}$ in the reactor. Figure 3(a) presents a
SEM cross section image of as grown undoped Si NWs whose diameter mean value is $50 \mathrm{~nm}$. Then, the NWs are oxide etched with an $\mathrm{HF}$ solution, and the $\mathrm{Au}$ catalyzer residues are suppressed with an IK: $\mathrm{I}_{2}$ solution. The NW array is then encapsulated by spin coating a solution of SOG (spin on glass) material on the substrate. The sample top surface is afterward submitted to a CMP (Chemical Mechanical Polishing) process to reduce the surface roughness and hence to facilitate the SThM scanning. A final etch is realized with an HF solution during a few seconds to ensure a good digging out of the nanowires. Figure 3(b) presents a SEM top view image of a Si NW sample after the encapsulation process. From this image, we can observe three NWs jutting out above the $\mathrm{SiO}_{2}$ die of a few tens of nanometers.

Two samples have been developed, the first one with undoped Si NWs and the second one with n doped Si NWs corresponding to a $2 \times 10^{-5}$ dopant to silicon ratio. Their diameter and length are, respectively, evaluated by SEM images to $49 \mathrm{~nm} \pm 6 \mathrm{~nm}$ and $1.10 \mu \mathrm{m} \pm 0.05 \mu \mathrm{m}$ for the undoped sample and $51 \mathrm{~nm} \pm 7 \mathrm{~nm}$ and $0.80 \mu \mathrm{m} \pm 0.05 \mu \mathrm{m}$ for the $\mathrm{n}$ doped one. Figure 3(c) and 3(d), respectively, present the 2 $\mu \mathrm{m} \times 3 \mu \mathrm{m}$ topographical image and the corresponding thermal image of the undoped NW sample obtained simultaneously by $3 \omega \mathrm{SThM}$ imaging under atmospheric conditions. The probe has been supplied with a $f=1 \mathrm{kHz}$ current, hence a $2 \mathrm{kHz}$ Joule thermal frequency, lower than its thermal cutoff frequency measured to be $2.75 \mathrm{kHz}$. The pixel acquisition time is then $5 \mathrm{~ms}$, which leads to a 4 min acquisition time since each image is constituted of $256 \times 170$ pixels. All the $3 \omega$ SThM images presented afterward are obtained with the same experimental conditions, except for the number of pixels constituting the images. The thermal exchange radius, due to the various thermal transfer mechanisms (radiation, solidsolid, air, and water meniscus conductions), has been measured $^{24}$ to be $b=100 \mathrm{~nm}$, so 4 times higher than the Si NW mean radius. That means that, as represented in Figure 4, the whole heat flux does not go through the Si NW, but a part of it crosses the silica die.

Since the $\mathrm{SiO}_{2}$ die thickness (about $1 \mu \mathrm{m}$ ) is ten times higher than the thermal exchange radius, its thermal resistance $R_{\mathrm{SiO} 2}$ can be evaluated as a constriction resistance using the Maxwell

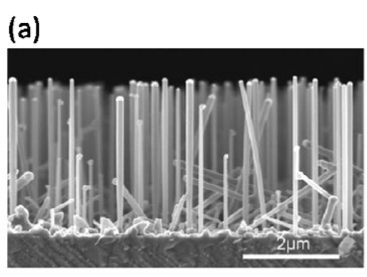

(c)

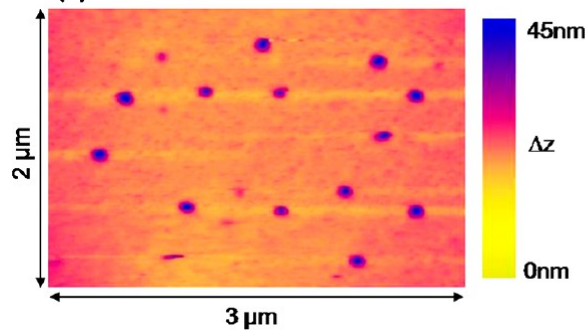

(b)

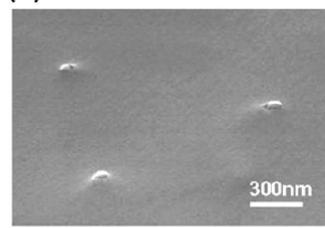

(d)

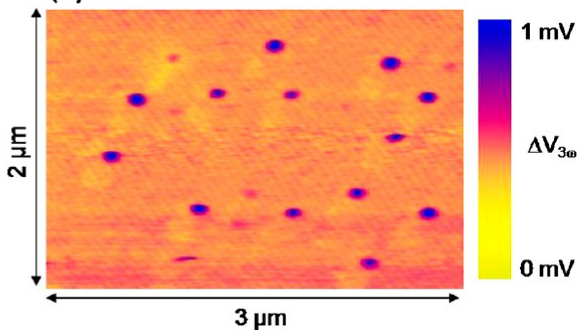

Figure 3. VLS Si nanowires: (a) cross section SEM image, (b) top view SEM image after the encapsulation process, (c) $2 \mu \mathrm{m} \times 3 \mu \mathrm{m}$ topographical image, and (d) $2 \mu \mathrm{m} \times 3 \mu \mathrm{m} 3 \omega$ thermal image. 

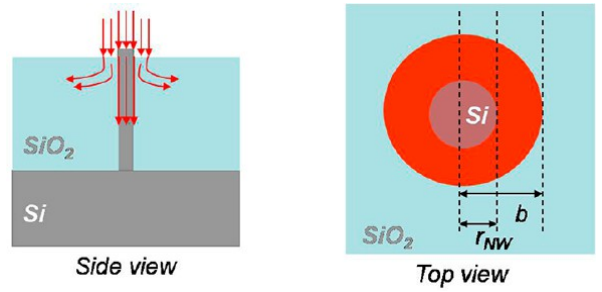

Figure 4. Modelization of the thermal flux applied to the VLS Si NW sample.

relation. ${ }^{28,29}$ This constriction resistance is due to the heat flux propagation through a $S_{\mathrm{SiO}_{2}}$ surface ring with an external radius $b$ and an internal radius $r_{\mathrm{NW}}$ (Figure 4) and can be expressed as

$$
R_{\mathrm{SiO}_{2}}=\frac{1}{4 \lambda_{\mathrm{SiO}_{2}} \sqrt{b^{2}-r_{\mathrm{NW}}^{2}}}
$$

where $\lambda_{\mathrm{SiO}_{2}}$ is the $\mathrm{SiO}_{2}$ thermal conductivity and $r_{\mathrm{NW}}$ the nanowire radius. With $\lambda_{\mathrm{SiO}_{2}}=1.3 \mathrm{~W} \mathrm{~m}^{-1} \mathrm{~K}^{-1} ; b=100 \mathrm{~nm}$; and $r_{\mathrm{NW}}=25 \mathrm{~nm}, R_{\mathrm{SiO}_{2}} \approx 2 \times 10^{6} \mathrm{~K} / \mathrm{W}$. We can compare it with the $\mathrm{NW}$ intrinsic thermal resistance for a diffusive heat transfer

$$
R_{\mathrm{NW}}=\frac{1}{\lambda_{\mathrm{NW}}} \frac{L}{S}=\frac{L}{\lambda_{\mathrm{NW}} \times \pi \times r_{\mathrm{NW}}{ }^{2}}
$$

where $\lambda_{\mathrm{NW}}$ is the nanowire thermal conductivity. Taking $\lambda_{\mathrm{NW}}=$ $25 \mathrm{~W} \mathrm{~m}^{-1} \mathrm{~K}^{-114}$ and $L=1 \mu \mathrm{m}, R_{\mathrm{NW}} \approx 2 \times 10^{7} \mathrm{~K} / \mathrm{W}$.

From these estimations, we note that, even if the tip is in direct contact with the emerging NW, the heat flux goes preferentially through the $\mathrm{SiO}_{2}$ mostly by water meniscus and air conduction and not through the $\mathrm{Si}$ NW. Under atmospheric conditions, we are then more sensitive to the thermal properties of the $\mathrm{SiO}_{2}$ than to those of the NW. We have experimentally noted that the $3 \omega$ signal, hence the equivalent thermal resistance, increases when the NW diameter increases. This trend can be explained by the fact that when the NW diameter increases the $S_{\mathrm{SiO}_{2}}$ surface decreases, and then the correspond ing equivalent thermal resistance increases, confirming the major role of the $\mathrm{SiO}_{2}$ die.

To be sensitive to the NW and perform quantitative measurements, it is therefore necessary to work under vacuum $\left(10^{-5}\right.$ Torr $)$ to reduce the thermal exchanges to the solid-solid conduction between the tip and the NWs. Under these experimental conditions, we have then deduced the mean equivalent thermal resistances for the undoped and $\mathrm{n}$ doped samples

$$
\begin{aligned}
& \left\langle R_{\text {eq }}\right\rangle_{\text {undoped }}=3.1 \times 10^{7} \mathrm{~K} / \mathrm{W} \pm 3 \times 10^{6} \mathrm{~K} / \mathrm{W} \\
& \left\langle R_{\text {eq }}\right\rangle_{\mathrm{n} \text {-doped }}=3.5 \times 10^{7} \mathrm{~K} / \mathrm{W} \pm 3 \times 10^{6} \mathrm{~K} / \mathrm{W}
\end{aligned}
$$

To deduce $\lambda_{\mathrm{NW}}$ from eq 2 , the next step consists of determining the various thermal resistances $R_{\text {Tip-NW}}, R_{\mathrm{NW} \text {-Sub }}$ and $R_{\mathrm{c}}$.

(i) $R_{\text {Tip-NW: }}$ the constriction resistance between the tip and the nanowire is negligible as the thermal exchange surface is limited by the NW section.

(ii) $R_{\mathrm{NW} \text {-Sub }}$ the constriction resistance between the NW and the substrate can be expressed as ${ }^{28}$

$$
R_{\mathrm{NW}-\mathrm{Sub}}=\frac{1}{2 \lambda_{\mathrm{Si}} d_{\mathrm{NW}}}
$$

where $\lambda_{\mathrm{Si}}$ is the thermal conductivity of the bulk substrate and $d_{\mathrm{NW}}$ the NW diameter. With $\lambda_{\mathrm{Si}}=150 \mathrm{~W} \mathrm{~m}^{-1} \mathrm{~K}^{-1}$ and the mean NW diameter $d_{\mathrm{NW}}=50 \mathrm{~nm}, R_{\mathrm{NW}-\mathrm{Sub}} \approx 6.7 \times 10^{4} \mathrm{~K} / \mathrm{W}$. This resistance is hence negligible compared with both mean equivalent thermal resistances. Then, $R_{\mathrm{eq}}=R_{\mathrm{c}}+R_{\mathrm{NW}}$.

(iii) The issue is now to determine the contact thermal resistance $R_{\mathrm{c}}$.

The contact resistance is often determined by calibration on a material of known thermal conductivity. ${ }^{19,30}$ It is then assumed that $R_{\mathrm{c}}$ does not change from sample to sample and when measuring other materials. Nevertheless, some precau tions need to be taken as this contact resistance is very dependent on various parameters such as the surface roughness or the tip to sample contact geometry. An alternative solution will be presented in the next section, but then a large dispersion on the NW diameters is necessary, which is not the case for our current Si NW sample. Then, to evaluate a range of values for the $\mathrm{Si}$ NW thermal conductivity, we have made two hypotheses: the optimistic one considering the contact thermal resistance as negligible and the pessimistic one considering it as predominant.

In the first case, the mean equivalent thermal resistance is then equal to the intrinsic thermal resistance of the NWs. From eq 4 , we hence deduce the minimum possible values of the thermal conductivity of the nanowires

$$
\begin{aligned}
& \left(\lambda_{\text {undoped }}\right)_{\min }=(18 \pm 4) \mathrm{W} \mathrm{m}^{-1} \mathrm{~K}^{-1} \text { for the undoped NWs } \\
& \left(\lambda_{\text {n-doped }}\right)_{\min }=(12 \pm 3) \mathrm{W} \mathrm{m}^{-1} \mathrm{~K}^{-1} \text { for the } \mathrm{n} \text {-doped NWs }
\end{aligned}
$$

The uncertainties are calculated taking into account the uncertainties on the equivalent thermal resistances, as well as the ones on the diameter and length of the NWS.

In the second hypothesis, since $R_{\mathrm{eq}}=R_{\mathrm{c}}+R_{\mathrm{NW}}$, considering $R_{\mathrm{c}}$ as predominant and constant from one NW to another, $R_{\mathrm{c}}$ cannot be higher than the smallest equivalent thermal resistance $R_{\mathrm{eq}}^{-}$measured on the different NWs of the thermal image. This constitutes the most pessimistic case for which we obtain the maximum possible NW thermal conductivity

$$
(\lambda)_{\max }=\frac{L}{S} \times \frac{1}{\left\langle R_{\mathrm{eq}}\right\rangle-R_{\mathrm{eq}}^{-}}
$$

The values are then

$$
\begin{aligned}
\left(\lambda_{\text {undoped }}\right)_{\max }= & (58 \pm 12) \mathrm{W} \mathrm{m}^{-1} \mathrm{~K}^{-1} \\
& \text { for the undoped NWs } \\
\left(\lambda_{\text {n-doped }}\right)_{\max }= & (39 \pm 9) \mathrm{W} \mathrm{m}^{-1} \mathrm{~K}^{-1} \text { for the } \mathrm{n} \text {-doped NWs }
\end{aligned}
$$

Even if we are not able to determine a precise value of the NW thermal conductivity, we note, in any case, a reduction in comparison with the bulk value. As the phonon mean free path (around $300 \mathrm{~nm}$ in $\mathrm{Si}$ at room temperature) is higher than the NW diameter, we expect a theoretical thermal conductivity reduction which is confirmed by our measurements. In addition, $\mathrm{Li}^{14}$ has measured a $25 \mathrm{~W} \mathrm{~m} \mathrm{~m}^{-1} \mathrm{~K}^{-1}$ thermal conductivity at $300 \mathrm{~K}$ for a $56 \mathrm{~nm}$ diameter $\mathrm{Si} \mathrm{NW}$, which is in the thermal conductivity range found for both undoped and $\mathrm{n}$ doped NWs. Nevertheless, we can only give an interval for the thermal conductivity value and not a precise value as we are not able to accurately measure the thermal contact resistance $R_{\mathrm{c}}$. Consequently, we propose in the next section an experimental method to obtain a reliable value of the thermal 
contact resistance and therefore of the thermal conductivity, but this method requires us to produce a set of NW samples with a wide range of diameters.

\section{THERMAL CONDUCTIVITY OF NANOWIRES ELABORATED BY CHEMICAL WET ETCHING}

4.1. Si NW Chemical Etching Process. The sample is an assembly of $\mathrm{Si}$ NWs embedded in a silica $\mathrm{SiO}_{2}$ die. The $\mathrm{Si}$ NWs have been made via a metal assisted chemical wet etching process. This method was introduced by $\mathrm{Li}^{31}$ in 2000 to produce porous silicon. This process named HOME HF for " $\underline{\mathrm{H}}_{2} \underline{\mathrm{O}}_{2}$ metal $\underline{\mathrm{HF}}$ etching" is a two step reaction that involves the predeposition of a metal thin film, followed by chemical etching in the presence of $\mathrm{HF}$ and $\mathrm{H}_{2} \mathrm{O}_{2}$. According to the authors, ${ }^{31}$ this etching reaction may occur as an electrochemical process localized around the nanometer sized metal film that acts as a catalyst. Lately, this method has been used to produce arrays of Si NWs by patterning the metal thin film. ${ }^{32,33}$ Here, we have developed the process exposed elsewhere ${ }^{32}$ to produce a dense vertically aligned array of Si NWs.

First, the metal film is patterned using nanosphere lithography. Indeed, a closed packed two dimensional array of $500 \mathrm{~nm}$ diameter silica colloids is used as a template. Successively sintering and etching the colloids enables us to turn the closed packed 2D array into a nonclosed packed one. Figure 5(a) presents a SEM top view image of a nonclosed
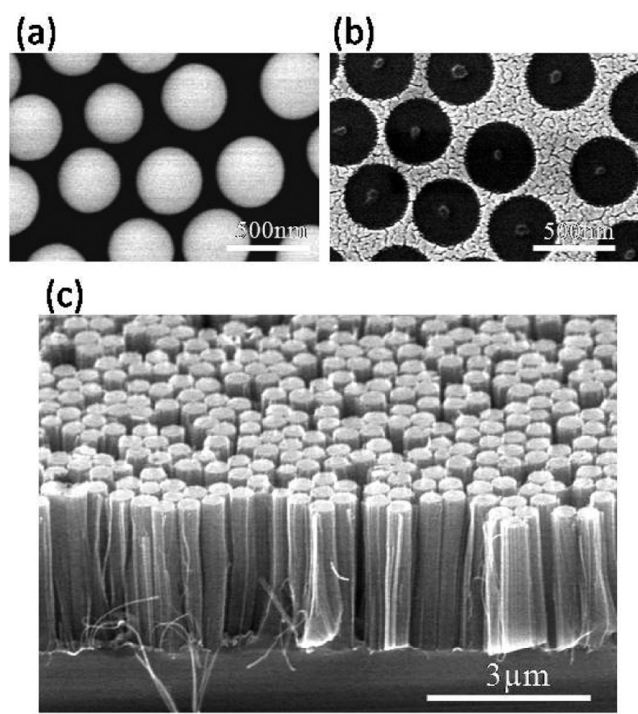

Figure 5. HOME HF process: SEM images of (a) a nonclosed packed 2D silica colloid array, (b) a patterned Au thin film, and (d) a wet etched Si NW array.

packed 2D silica colloid array which has been sintered at 1000 ${ }^{\circ} \mathrm{C}$ during $2 \mathrm{~h}$ and subsequently etched during $1 \mathrm{~min}$ in a $\mathrm{HF}$ solution. Thereafter, a $10 \mathrm{~nm}$ thick Au film is evaporated on the substrate, and the silica colloid template is lifted off by brief ultrasonication in deionized water for 2-3 min. Figure 5(b) presents a SEM image of a patterned Au thin film after the silica colloids lift off process. Then, the substrate is immersed in an $\mathrm{HF}(10 \%)-\mathrm{H}_{2} \mathrm{O}_{2}(0.6 \%)$ solution during $10 \mathrm{~min}$ to produce a dense array of $1.7 \mu \mathrm{m}$ long and $480 \mathrm{~nm}$ mean diameter Si NWs (Figure 5(c)). The $\mathrm{Au}$ catalyzer residues are subsequently suppressed with an $\mathrm{IK}-\mathrm{I}_{2}$ solution. To reduce the $\mathrm{NW}$ mean diameter which is actually defined by the silica colloids size, we thermally oxidize the array and etch the formed oxide shell. The superficial $\mathrm{NW}$ thermal oxidation is therefore performed under $\mathrm{O}_{2}$ at $900{ }^{\circ} \mathrm{C}$ during $2 \mathrm{~h}$. The etching of the formed oxide shell is realized in an HF solution. This operation of diameter reduction has been successively repeated twice.

Finally, the Si NW array is encapsulated by spin coating a solution of SOG (spin on glass) material on the substrate. The sample top surface is then submitted to a CMP (Chemical Mechanical Polishing) process to reduce the surface roughness and hence to facilitate the SThM scanning. Figure 6(a) is a SEM image of the obtained Si NW array. Figure 6(b) presents the final sample embedded in the $\mathrm{SiO}_{2}$ die.

This process, in comparison with the VLS process, enables us to control the size and spacing of the NWs. In our case, we have created a set of three samples with $\mathrm{Si}$ NW diameters ranging from 200 to $380 \mathrm{~nm}$ with a $290 \mathrm{~nm}$ mean value measured by SEM. Their length $L_{\mathrm{SiNW}}$ has also been measured by SEM: $L_{\mathrm{SiNW}}=1.700 \mu \mathrm{m} \pm 50 \mathrm{~nm}$. Moreover, the diameter of the NWs being bigger than that of the heat exchange surface, when the tip is positioned at the center of the NW, the heat flux will go through the NW and not through the silica die, and we can hence work under atmospheric conditions.

4.2. Thermal Conductivity of HOME-HF Si NWs. We carry out simultaneously both topographic and thermal images of the sample top surface (respectively, Figure 6(c) and 6(d)). Both of them are $5 \mu \mathrm{m} \times 5 \mu \mathrm{m}$ sized pictures, with a resolution of 256 pixels $\times 256$ pixels. By measuring the $V_{3 \omega}$ bridge voltage from image $6(\mathrm{~d})$ on several tens of nanowires, we have deduced the mean value $\left(R_{\mathrm{eq}}\right)_{\mathrm{SiNWs}}=(3.792 \pm 0.001) \times 10^{6} \mathrm{~K} / \mathrm{W}$. The various NWs have different diameters, so depending on the diameter, the measured equivalent thermal resistance varies from $3.660 \times 10^{6}$ to $3.949 \times 10^{6} \mathrm{~K} / \mathrm{W}$. As previously mentioned, we have

$$
\left(R_{\mathrm{eq}}\right)_{\mathrm{SiNW}}=R_{\mathrm{c}}+R_{\mathrm{Tip}-\mathrm{NW}}+R_{N W}+R_{\mathrm{NW}-\mathrm{Sub}}
$$

(i) $R_{\text {Tip-NW }}$ can be evaluated by the expression of the constriction resistance between a circular heat source and a semi infinite circular cylinder ${ }^{34}$

$$
R_{\mathrm{Tip}-\mathrm{NW}}=\frac{\psi}{\lambda_{\mathrm{NW}} \sqrt{\pi} b}
$$

where $\psi$ is a constriction adimensional parameter depending on the ratio between the heat radius and the NW radius; $\lambda_{\mathrm{NW}}$ is the thermal conductivity of the NW; and $b$ is the thermal exchange radius. Since we do not know the NW thermal conductivity, we cannot evaluate this resistance, but we can compare it to the intrinsic thermal resistance as the ratio between both resistances is given from eqs 4 and 8

$$
\frac{R_{\mathrm{Tip}-\mathrm{NW}}}{R_{\mathrm{NW}}}=\frac{\psi \sqrt{\pi} d_{\mathrm{NW}}^{2}}{4 L b}
$$

(ii) The other constriction resistance, namely, $R_{\mathrm{NW}-\mathrm{Sub}}$, can be evaluated by using eq 5 .

From eqs 5 and 9, we finally deduce that eq 7 can be rewritten as

$$
\left(R_{\mathrm{eq}}\right)_{\mathrm{SiNW}}=\left(\frac{1}{2 \lambda_{\mathrm{Si}} d_{\mathrm{NW}}}+R_{\mathrm{c}}\right)+R_{\mathrm{NW}} \times\left(1+\frac{\psi \sqrt{\pi} d_{\mathrm{NW}}^{2}}{4 L b}\right)
$$

In the case of the HOME HF Si NWs, with $\lambda_{\mathrm{Si}}=150 \mathrm{~W} \mathrm{~m}^{-1}$

$\mathrm{K}^{-1}$ and with a mean diameter $d_{\mathrm{NW}}=290 \mathrm{~nm}, R_{\mathrm{NW}-\mathrm{Sub}} \approx 1.15 \times$ 
(a)

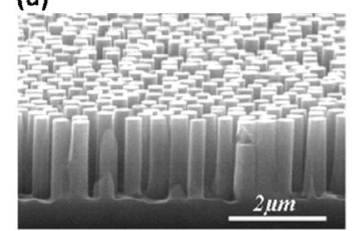

(c)

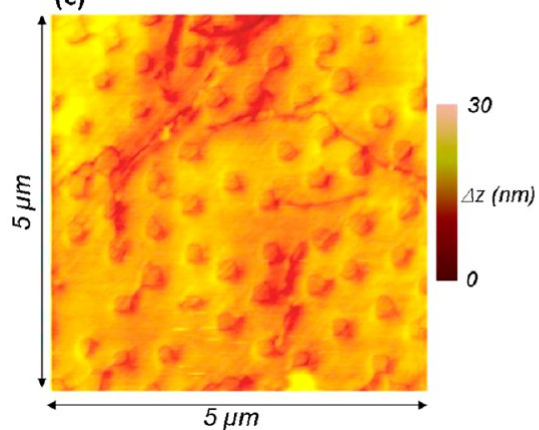

(b)

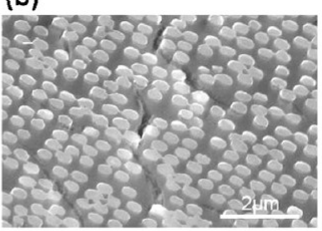

(d)

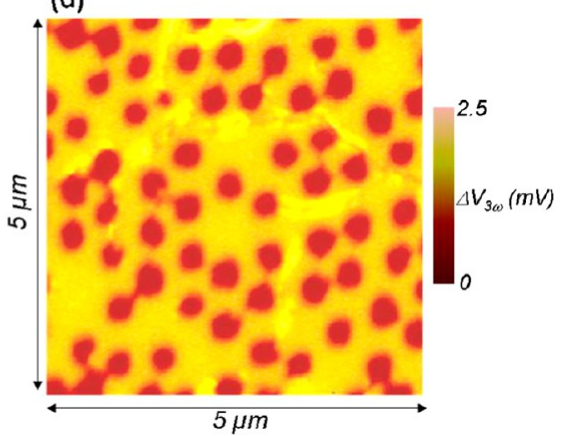

Figure 6. HOME HF Si nanowires: SEM images, respectively, before (a) and after (b) the encapsulation process, (c) $5 \mu \mathrm{m} \times 5 \mu \mathrm{m}$ topographical image, and (d) $5 \mu \mathrm{m} \times 5 \mu \mathrm{m}$ thermal image.

$10^{4} \mathrm{~K} / \mathrm{W}$. It is even lower than in the case of the VLS Si NWs, as the HOME HF NW diameter is bigger than the VLS NW one. In addition, with $L=1.7 \mu \mathrm{m}, b=100 \mathrm{~nm}$, and $\psi=0.084,{ }^{34}$ the $\left(\psi \sqrt{ } \pi d_{\mathrm{NW}}^{2}\right) /(4 L b)$ ratio value from eq 9 is $1.8 \times 10^{-2}$. Therefore, both $R_{\text {Tip-NW }}$ and $R_{\mathrm{NW} \text {-Sub }}$ are negligible.

(iii) Determination of $R_{\mathrm{c}}$ and $\lambda_{\mathrm{NW}}$. Considering $R_{\text {Tip-NW }}$ and $R_{\mathrm{NW}-\mathrm{Sub}}$ to be negligible, we can deduce from eqs 4 and 10 that the curve representing $R_{\text {eq }}$ as a function of the $L / S$ length to surface ratio of the NWs is a straight line whose $y$ intercept value gives the mean contact resistance and whose slope gives the NW thermal conductivity. This curve is presented for the Si NWs in Figure 7 with a best fit leading to a mean thermal

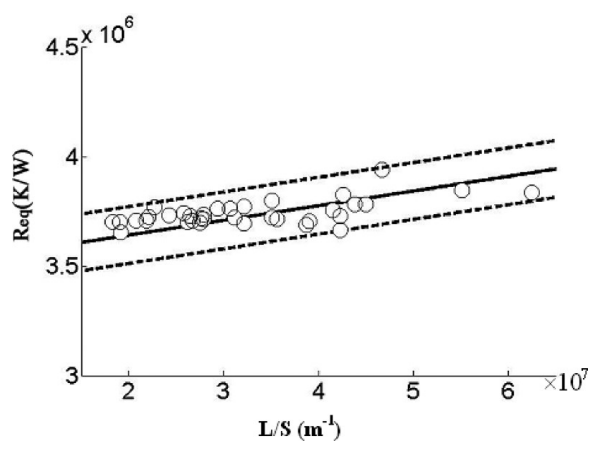

Figure 7. Si NW equivalent thermal resistance as a function of the ratio $L / S$ : circles are used for experimental data and lines for the fits.

conductivity $\lambda_{\mathrm{NW}}=148 \mathrm{~W} \mathrm{~m}^{-1} \mathrm{~K}^{-1}$ and a mean thermal contact resistance $R_{\mathrm{c}}=3.51 \times 10^{6} \mathrm{~K} / \mathrm{W}$. Both dashed curves take into account the dispersion on $R_{\mathrm{c}}$. These two curves enclose very well the experimental data when the incertitude on $R_{c}$ is set as $\left(\Delta R_{\mathrm{c}}\right) /\left(R_{\mathrm{c}}\right)=4 \%$. We can finally deduce $\lambda_{\mathrm{NW}}=(148 \pm 6) \mathrm{W}$ $\mathrm{m}^{-1} \mathrm{~K}^{-1}$ and $R_{\mathrm{c}}=(3.51 \pm 0.13) \times 10^{6} \mathrm{~K} / \mathrm{W}$.

To evaluate $R_{\mathcal{c}}$ a second method, which is usually done, consists of performing a measurement on a centimetric area of the sample which was voluntary not etched, consequently considered as a Si bulk area. Here, we assume that the surface state is the same on the Si bulk area and on Si NWs because these two different parts are located on the same sample. ${ }^{19,30}$ As a result, the tip to sample contact resistance can be considered as the same in both cases. Hence, we have measured on the $\mathrm{Si}$ bulk area a mean equivalent thermal resistance $\left(R_{\mathrm{eq}}\right)_{\mathrm{BulkSi}}=$ $(3.624 \pm 0.008) \times 10^{6} \mathrm{~K} / \mathrm{W}$ with

$$
\left(R_{\mathrm{eq}}\right)_{\mathrm{BulkSi}}=R_{\mathrm{c}}+R_{\mathrm{Tip}-\mathrm{Sub}}+R_{\mathrm{Bulk}}
$$

The Si substrate intrinsic thermal resistance $R_{\text {Bulk }}$ is negligible because the $\mathrm{Si}$ substrate section is very large. To evaluate $R_{\text {Tip-Sub, }}$ we consider a circular constriction defined by the tip to sample thermal exchange surface

$$
R_{\mathrm{Tip}-\mathrm{Sub}}=\frac{1}{4 \lambda_{\mathrm{BulkSi}} b}
$$

where $b$ is the radius of the circular thermal constriction. With $b$ $=100 \mathrm{~nm}$ and $\lambda_{\text {BulkSi }}=150 \mathrm{~W} \mathrm{~m}^{-1} \mathrm{~K}^{-1}$, we can evaluate $R_{\text {Tip-Sub }}$ and then deduce $R_{\mathrm{c}}=(3.60 \pm 0.01) \times 10^{6} \mathrm{~K} / \mathrm{W}$. We note a value which is quite similar to the mean value identified by the first method, the difference being less than 3\%, probably because the surface roughness is quite homogeneous due to the final surface polishing.

Considering this set of samples, we have taken advantage of the dispersion of the diameters of the Si NWs to do a statistical data processing. Our $3 \omega \mathrm{SThM}$ imaging method shows its relevance and interest since a single image can lead to a reliable estimation of both thermal contact resistance $R_{c}$ and thermal conductivity $\lambda_{\mathrm{NW}}$ mean values and uncertainties. The large diameter of the NWs is an advantage since we can work under atmospheric conditions but also a drawback since the phonon mean free path is of the same order than the NW diameter which implies no significant reduction of the conductivity. Consequently, the study of SiGe NWs with large diameters could be promising in terms of thermal conductivity reduction since, in this case, alloy scattering is the dominant scattering mechanism. The NW diameter reduction also contributes to the thermal conductivity reduction, but the thermal con ductivity dependency on the NW diameter is not significant in comparison with the one observed for Si NWs. ${ }^{14,35,36}$

4.3. Thermal Conductivity of HOME-HF SiGe NWs. SiGe NWs have therefore been fabricated using the HOME HF process described before for the Si NWs instead of the classical 
(a)

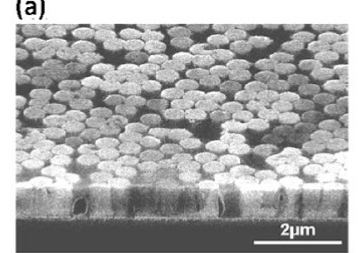

(c)

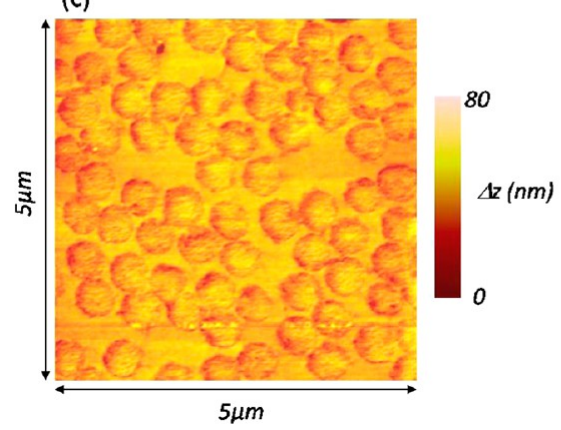

(b)
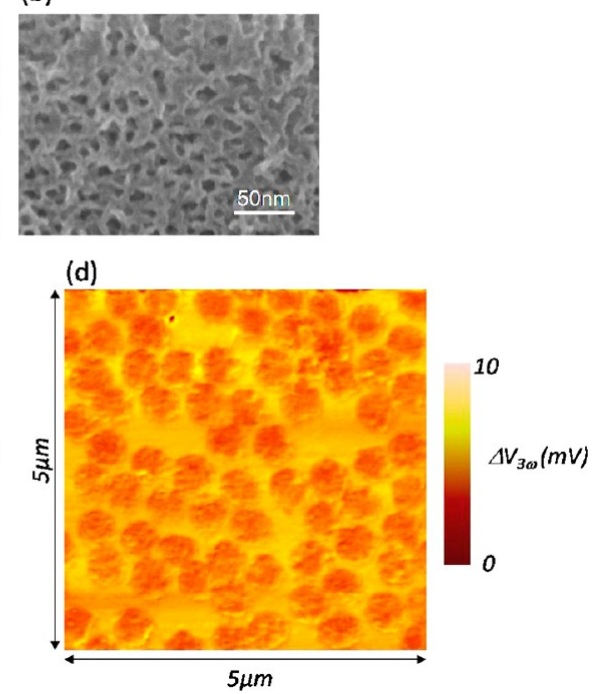

Figure 8. HOME HF SiGe nanowires: SEM images (a) of the encapsulated NW array, (b) within the core of one SiGe NW, (c) $5 \mu \mathrm{m} \times 5 \mu \mathrm{m}$ topographical image, and (d) $5 \mu \mathrm{m} \times 5 \mu \mathrm{m}$ thermal image.

VLS growth process. ${ }^{37}$ First, a $1 \mu \mathrm{m}$ thick SiGe epitaxial layer is deposited on a $\mathrm{Si}$ substrate by chemical vapor deposition under $\mathrm{SiH}_{4}$ and $\mathrm{GeH}_{4}$ vapor flow. The ratio of $\mathrm{GeH}_{4}$ and $\mathrm{SiH}_{4}$ flows is controlled to obtain a $10 \% \mathrm{Ge}$ concentration in the final $\mathrm{SiGe}$ thin film. Then, such as for Si NWs, a two dimensional array of $500 \mathrm{~nm}$ diameter silica colloids is used as a template. The colloids are successively sintered and etched to turn the closed packed $2 \mathrm{D}$ array into a nonclosed packed one. Then, a $10 \mathrm{~nm}$ thick $\mathrm{Au}$ film is evaporated on the substrate. The silica colloid template is lifted off by brief ultrasonication in deionized water. Finally, the chemical wet etching of $\mathrm{SiGe} \mathrm{NWs}$ is performed by immersing the substrate in a $\mathrm{HF}(10 \%)-\mathrm{H}_{2} \mathrm{O}_{2}(0.6 \%)$ solution during $20 \mathrm{~min}$. The as obtained $\mathrm{SiGe} \mathrm{NW}$ array is then encapsulated by spin coating a solution of SOG (spin on glass) material on the substrate, and the sample top surface is polished to reduce the surface roughness. Figure 8(a) presents a SEM image of the encapsulated SiGe NW array. The SiGe NW average diameter and length are then measured to be $d_{\mathrm{SiGe}}=$ $(480 \pm 20) \mathrm{nm}$ and $L_{\mathrm{SiGe}}=(750 \pm 50) \mathrm{nm}$, respectively. It should be noted that these NWs present a strong surface porosity which can be evaluated to $40 \%$ from Figure $8(\mathrm{~b})$. Contrary to the previous case concerning the elaboration of pure Si NWs, Ge impurities would probably serve here as nucleation sites for pore formation during the wet etching process. Because of this porosity, we could not reduce the diameters of the NWs.

The $5 \mu \mathrm{m} \times 5 \mu \mathrm{m}$ topographical and $3 \omega$ SThM thermal images are, respectively, presented in Figure $8(\mathrm{c})$ and $8(\mathrm{~d})$. They are constituted of $256 \times 256$ pixels. The topographical image shows the SiGe NWs jutting out above the die surface. In the thermal image, the local conductance increases when the heat flux is directly applied on a SiGe NW, but the signal is not homogeneous on the whole surface of the same NW. As this sample presents a strong porosity, this can be due to the pores which increase the roughness of the sample. To obtain a mean value of the thermal signal on each NW, we present in Figure 9 the statistical distribution of the $3 \omega$ voltage signal on the thermal image. We can note two maxima, the first one corresponding to the signal measured on the SiGe NWs and the second one corresponding to the silica die. If we consider

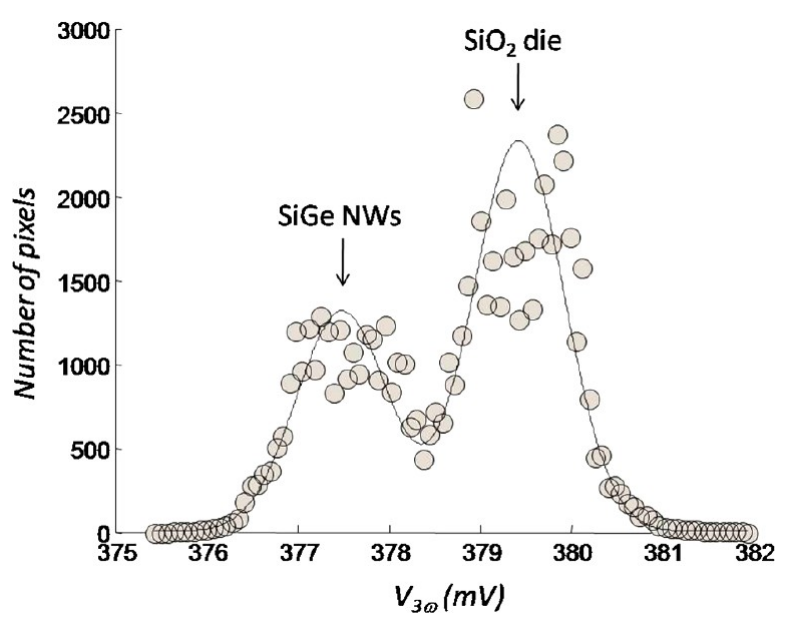

Figure 9. Statistical distribution of the $3 \omega$ voltage measured on the HOME HF SiGe NW sample.

the latter signal, we have measured on the $\mathrm{SiO}_{2}$ die a mean $3 \omega$ voltage $\left(V_{3 \omega}\right)_{\mathrm{SiO}_{2}}=(379.4 \pm 0.3) \mathrm{mV}$ which leads to an equivalent thermal resistance $\left(R_{\mathrm{eq}}\right)_{\mathrm{SiO}_{2}}=(6.35 \pm 0.05) \times 10^{6}$ $\mathrm{K} / \mathrm{W}$. This equivalent thermal resistance corresponds to the combination of a contact resistance $R_{\mathrm{c}}$ and of the tip to sample constriction resistance

$$
\left(R_{\mathrm{eq}}\right)_{\mathrm{SiO}_{2}}=R_{\mathrm{c}}+R_{\mathrm{Tip}^{-} \mathrm{SiO}_{2}}
$$

For the constriction resistance, we consider a circular constriction defined by the tip to sample thermal exchange surface to calculate $R_{\text {Tip-SiO2 }}$ and described by expression 12 with the $\mathrm{SiO}_{2}$ thermal conductivity $\lambda_{\mathrm{SiO} 2}$ instead of $\lambda_{\mathrm{BulkSi}}$. The radius of the circular thermal constriction has been measured to be $b=120 \mathrm{~nm}$. Since this sample does not offer a large diameter dispersion, the contact resistance has been evaluated by the mean value measured on a not etched area, using the second method previously described for the Si NWs, $R_{c}=(4.75$ $\pm 0.01) \times 10^{6} \mathrm{~K} / \mathrm{W}$. We can hence deduce the thermal conductivity of the silica die 


$$
\lambda_{\mathrm{SiO}_{2}}=\frac{1}{4 b\left(\left(R_{\mathrm{eq}}\right)_{\mathrm{SiO}_{2}}-R_{\mathrm{c}}\right)}=(1.30 \pm 0.04) \mathrm{W} \mathrm{m}^{-1} \mathrm{~K}^{-1}
$$

This value is in excellent agreement with values reported in the literature, ${ }^{38,39}$ which is a good indicator of the validity of the $R_{c}$ identified value. From the first maximum in Figure 9, we can finally deduce the mean thermal conductivity of the SiGe NWs. Measuring the $V_{3 \omega}$ bridge voltage on several tens of nanowires of the thermal image (Figure $8(\mathrm{~d})$ ), we have indeed deduced the mean value $\left(R_{\mathrm{eq}}\right)_{\mathrm{SiGeNWs}}=(6.05 \pm 0.07) \times 10^{6} \mathrm{~K} / \mathrm{W}$.

Then, from eqs 7 and 9 , we can deduce

$$
\left(R_{\mathrm{eq}}\right)_{\mathrm{SiGeNWs}}=R_{\mathrm{NW}}\left(1+\frac{\psi \sqrt{\pi} d_{\mathrm{NW}}^{2}}{4 L b}\right)+R_{\mathrm{c}}+R_{\mathrm{NW}-\mathrm{Sub}}
$$

Using eq $5, R_{\mathrm{NW}-\text { Sub }}$ can be evaluated to $8 \times 10^{3} \mathrm{~K} / \mathrm{W}$, hence negligible and then

$$
\lambda_{\mathrm{NW}} \approx \frac{L}{S}\left[\frac{\left(1+\frac{\psi \sqrt{\pi} d_{\mathrm{NW}}^{2}}{4 L b}\right)}{\left(R_{\mathrm{eq}}\right)_{\mathrm{SiGeNWs}}-R_{\mathrm{c}}}\right]
$$

The SiGe NW mean thermal conductivity is then evaluated to $(3.8 \pm 0.2) \mathrm{W} \mathrm{m}^{-1} \mathrm{~K}^{-1}$, far lower than the $22 \mathrm{~W} \mathrm{~m}^{-1} \mathrm{~K}^{-1}$ bulk value for $\mathrm{Si}_{0.9} \mathrm{Ge}_{0.1}$. The uncertainty is calculated taking into account the uncertainties on $\left(R_{\mathrm{eq}}\right)_{\mathrm{SiGeNW}}, R_{\mathcal{O}} L_{\mathrm{SiGe}}$ and $d_{\mathrm{SiGe}}$. These SiGe NWs seem to be very good candidates for thermoelectric applications. Indeed, even if their diameter is large, which makes the measurements easier, their thermal conductivity is highly reduced in comparison with bulk SiGe, and in addition, their thermoelectric power factor $S^{2} \sigma$ should be only slightly reduced. ${ }^{40}$ Here, because of the homogeneity of the NW diameters, we have taken for $R_{c}$ the mean value measured on different positions of the silica die. We have seen on the sample made of $\mathrm{Si}$ NWs that this method has given a value close to the one estimated by a statistical data processing which demands a wide dispersion of the NW diameters. The next step could consist of creating a SiGe NW sample with a wide dispersion of diameters to confirm our value of $R_{c}$ and therefore of $\lambda_{\mathrm{NW}}$. Nevertheless, the good estimation of $\lambda_{\mathrm{SiO} 2}$ let us assume that $R_{\mathrm{c}}$ has been correctly evaluated.

4.4. Discussion. We finally compare (Figure 10) the results presented in the previous sections with results reported in the literature. The $x$ axis corresponds to the critical dimension, which is defined as the diameter for circular NWs, and $d_{\mathrm{c}}=$ $(2 \sqrt{ } w t) /(\sqrt{ } \pi)$ for NWs with a rectangular section where $w$ is the width and $t$ the thickness of the NW. The curve for Si NWs corresponds to the boundary scattering model based on Matthiessen's rule for multiple scattering ${ }^{4}$

$$
\lambda_{\mathrm{NW}}=\frac{\lambda_{\text {BulkSi}} \times d_{\mathrm{c}}}{l_{\text {BulkSi }}+d_{\mathrm{c}}}
$$

where $l_{\text {Bulksi }}$ is the effective mean free path, which is around 300 $\mathrm{nm}$ at $300 \mathrm{~K}$. For the Si NWs whose diameter is lower than 100 $\mathrm{nm}$, our results are compared with experimental results obtained by Raman ${ }^{41}$ and microfabricated suspended device techniques. ${ }^{9,14}$ All the measurements fit quite well with the boundary scattering model and show a reduction of the thermal conductivity as the Si NW diameter decreases. Indeed, the phonon mean free path in $\mathrm{Si}$ at room temperature is higher than the NW diameter, and the reduction of thermal conductivity can then be attributed to boundary scattering of

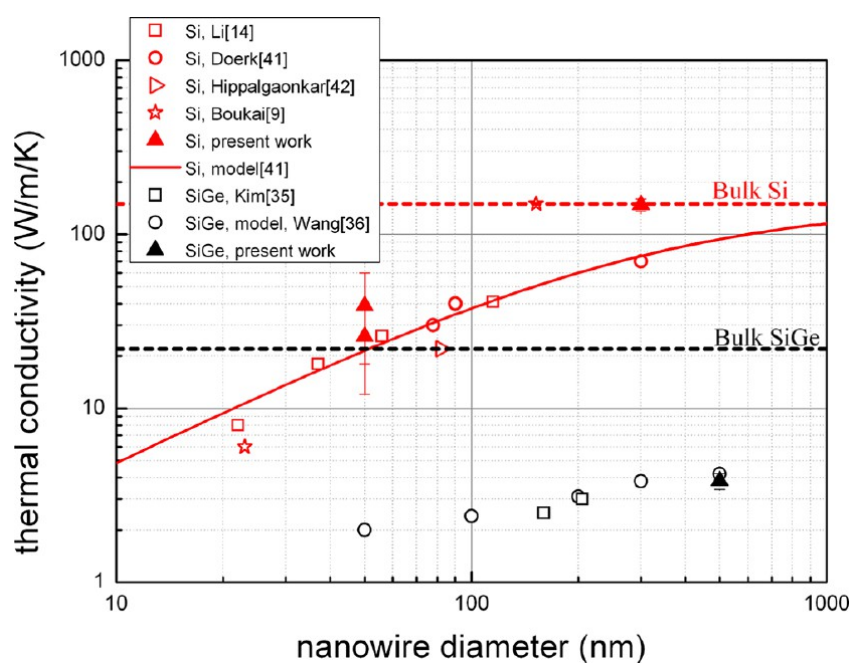

Figure 10. Thermal conductivity measurements on individual $\mathrm{Si}$ and SiGe NWs by Raman, microsuspended device, and $3 \omega$ SThM techniques as well as predicted values by boundary scattering models. The dotted lines indicate the $\mathrm{Si}$ (red line) and SiGe (black line) bulk thermal conductivities.

phonons. The values we could identify by $3 \omega$ SThM measurements, whether it be for nondoped or $\mathrm{n}$ doped $\mathrm{Si}$ NWs, are in the range of values obtained by the other experimental methods and fit quite well the boundary scattering model curve. A recent measurement for $82 \mathrm{~nm}$ EBL (Electron Beam Lithography) based integrated Si NWs leads to a value slightly lower than the one obtained for VLS Si NWs. ${ }^{42}$ The authors have explained it by the higher roughness which increases the phonon scattering mechanism and then reduces again the thermal conductivity.

As for the large diameter Si NWs, our measurements do not exhibit a significant reduction of the thermal conductivity, which can be explained by the fact that the NW diameter approaches the bulk Si phonon mean free path at $300 \mathrm{~K}$. As a consequence, the phonon boundary scattering phenomenon mentioned elsewhere ${ }^{8,12-14}$ is not the most dominant scattering mechanism controlling the thermal transport in our Si NWs. Nevertheless, according to the boundary scattering model or Raman measurements, ${ }^{41}$ a value around $80 \mathrm{~W} \mathrm{~m}^{-1}$ $\mathrm{K}^{-1}$ should be expected, hence lower than our $148 \mathrm{~W} \mathrm{~m}^{-1} \mathrm{~K}^{-1}$ value. However, Hochbaum et al. ${ }^{8}$ have emphasized the fact that the roughness at the $\mathrm{Si} N \mathrm{NW}$ surface may strongly reduce the thermal conductivity. In our precise case, the Si NWs have been thermally oxidized to reduce their diameter. This operation of diameter reduction is known to produce a very smooth surface which does not contribute to reduce the thermal conductivity. In addition, the thermal conductivity of $\mathrm{Si}$ nanowires with a $520 \mathrm{~nm} \times 35 \mathrm{~nm}$ cross section, hence a section smaller than our Si NWs, and a critical dimension $d_{c}=$ $152 \mathrm{~nm}$, smaller than the NW diameter of our sample, has been measured by Boukai $^{9}$ to be identical to the bulk value (red dotted line in Figure 10).

Concerning the SiGe NWs, let us first underline that there exist very few results for SiGe NW thermal conductivity measurements. The thermal conductivity obtained by $3 \omega$ SThM measurements on SiGe NWs is here compared with the value measured using the microsuspended device ${ }^{35}$ and with values obtained by a theoretical model based on alloy and boundary scattering contributions. ${ }^{36}$ Indeed, in the case of SiGe 
NWs, high frequency phonons are scattered by the Ge atoms which behave as impurities, in addition to the low frequency phonon boundary scattering. Both scattering mechanisms lead to a thermal conductivity reduction, which occurs even for diameters as large as a few hundreds of nanometers since alloy scattering is the dominant scattering mechanism. ${ }^{14,35,36}$ This thermal conductivity reduction is observed for all the measurements, whatever the method and the NW diameter.

In our case, we observe a large reduction of thermal conductivity in comparison with the $22 \mathrm{~W} \mathrm{~m}^{-1} \mathrm{~K}^{-1}$ bulk value (black dotted line in Figure 10). This can be partly ascribed to the high porosity of our NWs, which can considerably reduce the thermal conductivity. In our case, the porosity has been evaluated to around $40 \%$. The thermal conductivity reduction due to the porosity can be evaluated using the classic Eucken model $\lambda_{\text {porous }} / \lambda_{\text {solid }}=(1-\varphi) /(1+\varphi / 2)$ or the Russell model $\lambda_{\text {porous }} / \lambda_{\text {solid }}=\left(1-\varphi^{2 / 3}\right) /\left(1-\varphi^{2 / 3}+\varphi\right)$ where $\varphi$ is the porosity. ${ }^{43}$ Both models, respectively, lead to a $50 \%$ and $53 \%$ thermal conductivity reduction. Taking into account this porosity, the thermal conductivity should be reduced from the $22 \mathrm{~W} \mathrm{~m}^{-1} \mathrm{~K}^{-1}$ bulk value to about $11 \mathrm{~W} \mathrm{~m}^{-1} \mathrm{~K}^{-1}$. The value we have identified is 3 times lower. Nevertheless, it is in very good agreement with the value expected from the model described in ref 36 . In addition, Tang et al. ${ }^{40}$ have already observed the same tendency on holey silicon structures for which the reduction of thermal conductivity due to the porosity should lead to a $42 \mathrm{~W} \mathrm{~m}^{-1} \mathrm{~K}^{-1}$ thermal conductivity according to the Eucken model, while they measure values around $10 \mathrm{~W}$ $\mathrm{m}^{-1} \mathrm{~K}^{-1}$ or lower, depending on the dimensions of the structures (pitch and neck sizes). Hence, the porosity itself explains some of the reduction but does not capture the full reduction as underlined by several studies on porous solids: ${ }^{40,43}$ apart from the porous nature of the solid, the supplementary thermal conductivity can be ascribed to the low frequency phonon scattering off the pore boundaries.

\section{CONCLUSION}

Three kinds of NW samples have been grown, and their thermal conductivity has been evaluated using a $3 \omega$ SThM imaging technique: scanning an assembly of individual NWs, we can deduce the mean value of the NW thermal conductivity from a statistical data processing. This determination demands the evaluation of three thermal resistances: two constriction resistances and the thermal contact resistance between the tip and NW. Their values depend on the experimental conditions, in particular on the NW diameter and on the thermal exchange diameter between the tip and sample. Each new sample or tip therefore demands a new calibration of these resistances. We have proposed various means adapted to the different NW samples to determine the thermal contact resistance between the tip and NW. In addition, we have underlined that a dispersion on the NW diameters can be a powerful tool to deduce an accurate value of this contact resistance.

We have shown a diameter effect on the thermal conductivity of Si NWs. A significant thermal conductivity reduction is observed for $50 \mathrm{~nm}$ diameter Si NWs, but the measurement procedure is then quite delicate. On the contrary, SiGe NWs offer a large reduction of their thermal conductivity even for diameters as large as $480 \mathrm{~nm}$, hence easier to fabricate and to characterize. This reduction is partially explained by the porous nature of the material and partially by the low frequency phonon scattering off the pore boundaries.
Consequently, SiGe NWs seem to be better candidates for thermoelectricity applications than Si NWs. The next step could consist of creating a set of SiGe NW samples with a wide dispersion of diameters and in evaluating not only their thermal conductivity but also their electrical conductivity and Seebeck coefficient to deduce their thermoelectric figure of merit ZT.

\section{AUTHOR INFORMATION}

\section{Corresponding Author}

*E mail: stephane.grauby@u bordeaux1.fr. Tel.: 33 (0)5 4000 2786. Fax: 33 (0)5 40006970.

\section{Notes}

The authors declare no competing financial interest.

\section{ACKNOWLEDGMENTS}

This work has been supported by the ANR PNANO. The authors thank S. Poncet, C. Morin, P. Faucherand, M. Levis, J. Gavillet, D. Hauser, and V. Remondière from CEA for the sample fabrication assistance.

\section{REFERENCES}

(1) Patino Lopez, L. D.; Dilhaire, S.; Grauby, S.; Salhi, M. A.; Ezzahri, Y.; Claeys, W.; Batsale, J. C. Characterization of Thermoelectric Devices by Laser Induced Seebeck ElectroMotive Force (LIS EMF) Measurement. J. Phys. D: Appl. Phys. 2005, 38, 1489-1497.

(2) Wang, W.; Zhang, G.; Li, X. Manipulating Growth of Thermoelectric Bi2Te3/Sb Multilayered Nanowire Arrays. J. Phys. Chem C 2008, 112, 15190-15194.

(3) Shirato, N.; Strader, J.; Kumar, A.; Vincent, A.; Zhang, P.; Karakoti, A.; Nacchimuthu, P.; Cho, H. J.; Seal, S.; Kalyanaraman, R. Thickness Dependent Self Limiting 1 D Tin Oxide Nanowire Arrays by Nanosecond Pulsed Laser Irradiation. Nanoscale 2011, 3, 10901101.

(4) Feng, X.; Huang, X.; Wang, X. Thermal Conductivity and Secondary Porosity of Single Anatase TiO2 Nanowire. Nanotechnology 2012, 23, 185701.

(5) Kim, P.; Shi, L.; Majumdar, A.; McEuen, P. L. Thermal Transport Measurements of Individual Multiwalled Nanotubes. Phys. Rev. Lett. 2001, 87, 2155021-2155024.

(6) Munoz Rojo, M.; Grauby, S.; Rampnoux, J. M.; Caballero Calero, O.; Martin Gonzalez, M.; Dilhaire, S. Fabrication of Bi2Te3 Nanowire Arrays and Thermal Conductivity Measurement by $3 \omega$ Scanning Thermal Microscopy. J. Appl. Phys. 2013, 113, 0543081-0543087.

(7) Chen, X.; Wang, Y.; Ma, Y.; Cui, T.; Zou, G. Origin of the High Thermoelectric Performance in Si Nanowires: A First Principle Study. J. Phys. Chem. C 2009, 113, 14001-14005.

(8) Hochbaum, A. I.; Chen, R.; Diaz Delgado, R.; Liang, W.; Garnett, E. C.; Najarian, M.; Majumdar, A.; Yang, P. Enhanced Thermoelectric Performance of Rough Silicon Nanowires. Nature 2008, 451, 163167.

(9) Boukai, A. I.; Bunimovich, Y.; Tahir Kheli, J.; Yu, J. K.; Goddard, W. A., III; Heath, J. R. Silicon Nanowires as Efficient Thermoelectric Materials. Nature 2008, 451, 168-171.

(10) Bandaru, P. R.; Pichanusakorn, P. An Outline of the Synthesis and Properties of Silicon Nanowires. Semicond. Sci. Technol. 2010, 25, 024003.

(11) Volz, S.; Chen, G. Molecular Dynamics Simulation of Thermal Conductivity of Silicon Nanowires. Appl. Phys. Lett. 1999, 75, 20562058.

(12) Mingo, N. Calculation of Si Nanowire Thermal Conductivity Using Complete Phonon Dispersion Relations. Phys. Rev. B 2003, 68, 1133081-1133084.

(13) Lacroix, D.; Joulain, K.; Terris, D.; Lemonnier, D. Monte Carlo Simulation of Phonon Confinement in Silicon Nanostructures: Application to the Determination of the Thermal Conductivity of Silicon Nanowires. Appl. Phys. Lett. 2006, 89, 1031041-1031043. 
(14) Li, D.; Wu, Y.; Kim, P.; Shi, L.; Yang, P.; Majumdar, A. Thermal Conductivity of Individual Silicon Nanowires. Appl. Phys. Lett. 2003, 83, 2934-2936.

(15) Shi, L.; Li, D.; Yu, C.; Jang, W.; Kim, D.; Yao, Z.; Kim, P.; Majumdar, A. Measuring Thermal and Thermoelectric Properties of One Dimensional Nanostructures using a Microfabricated Device. J. Heat Transfer 2003, 125, 881-888.

(16) Persson, A. I.; Koh, Y. K.; Cahill, D. G.; Samuelson, L.; Linke, H. Thermal Conductance of InAs Nanowire Composites. Nano Lett. 2009, 9, 4484.

(17) Puyoo, E.; Grauby, S.; Rampnoux, J. M.; Rouvière, E.; Dilhaire, S. Scanning Thermal Microscopy of Individual Silicon Nanowires. J. Appl. Phys. 2011, 109, 0243021-0243029.

(18) Shi, L.; Zhou, J.; Kim, P.; Bachtold, A.; Majumdar, A.; McEuen, P. L. Thermal Probing of Energy Dissipation in Current Carrying Carbon Nanotubes. J. Appl. Phys. 2009, 105, 1043061-1043065.

(19) Hinz, M.; Marti, O.; Gotsmann, B.; Lantz, M. A.; Dürig, U. High Resolution Vacuum Scanning Thermal Microscopy of $\mathrm{HfO} 2$ and $\mathrm{SiO} 2$. Appl. Phys. Lett. 2008, 92, 0431221-0431223.

(20) Cahill, D. G. Thermal Conductivity Measurement from 30 to 750 K: The $3 \omega$ Method. Rev. Sci. Instrum. 1990, 61, 802-808.

(21) Shi, L.; Plyasunov, S.; Bachtold, A.; McEuen, P. L.; Majumdar, A. Scanning Thermal Microscopy of Carbon Nanotubes Using Batch Fabricated Probes. Appl. Phys. Lett. 2000, 77, 4295-4297.

(22) Lefevvre, S.; Volz, S. $3 \omega$ Scanning Thermal Microscope. Rev. Sci. Instrum. 2005, 76, 0337011-0337016.

(23) Lefevvre, S.; Volz, S.; Saulnier, J. B.; Fuentes, C.; Trannoy, N. Thermal Conductivity Calibration for Hot Wire Based DC Scanning Thermal Microscopy. Rev. Sci. Instrum. 2003, 74, 2418-2423.

(24) Puyoo, E.; Grauby, S.; Rampnoux, J. M.; Rouvière, E.; Dilhaire, S. Thermal Exchange Radius Measurement: Application to Nanowire Thermal Imaging. Rev. Sci. Instrum. 2010, 81, 0737011-0737015.

(25) Westwater, J.; Gosain, D. P.; Tomiya, S.; Usui, S.; Ruda, H. Growth of Silicon Nanowires via Gold/Silane Vapor Liquid Solid Reaction. J. Vac. Sci. Technol. B 1997, 15, 554-557.

(26) Yu, J. Y.; Chung, S. W.; Heath, J. R. Silicon Nanowires: Preparation, Device Fabrication, and Transport Properties. J. Phys. Chem. B 2000, 104, 11864-11870.

(27) Celle, C.; Mouchet, C.; Rouvière, E.; Simonato, J. P.; Mariolle, D.; Chevalier, N.; Brioude, A. Controlled in Situ n Doping of Silicon Nanowires during VLS Growth and their Characterization by Scanning Spreading Resistance Microscopy. J. Phys. Chem. C 2010, 114, 760765.

(28) Prasher, R. S.; Phelan, P. E. Microscopic and Macroscopic Thermal Contact Resistances of Pressed Mechanical Contacts. J. Appl. Phys. 2006, 100, 0635381-0635388.

(29) Prasher, R. S. Predicting the Thermal Resistance of Nanosized Constrictions. Nano Lett. 2005, 5, 2155-2159.

(30) Zhang, Y.; Hapenciuc, C. L.; Castillo, E. E.; Borca Tasciuc, T.; Mehta, R. J.; Karthik, C.; Ramanath, G. A Microprobe Technique for Simultaneously Measuring Thermal Conductivity and Seebeck Coefficient of Thin Films. Appl. Phys. Lett. 2010, 96, 06210710621073.

(31) Li, X.; Bohn, P. W. Metal Assisted Chemical Etching in HF/ H2O2 Produces Porous Silicon. Appl. Phys. Lett. 2000, 77, 25722574.

(32) Peng, K.; Zhang, M.; Lu, A.; Wong, N. B.; Zhang, R.; Lee, S. T. Ordered Silicon Nanowire Arrays via Nanosphere Lithography and Metal Induced Etching. Appl. Phys. Lett. 2007, 90, 1631231-1631233.

(33) Huang, Z.; Zhang, X.; Reiche, M.; Liu, L.; Lee, W.; Shimizu, T.; Senz, S.; Gösele, U. Extended Aarrays of Vertically Aligned Sub $10 \mathrm{~nm}$ Diameter [100] Si Nanowires by Metal Assisted Chemical Etching. Nano Lett. 2008, 8, 3046-3051.

(34) Negus, K. J.; Yovanovich, M. M.; Beck, J. V. On the Nondimensionalization of Constriction Resistance for Semi Infinite Heat Flux Tubes. J. Heat Transfer 1989, 111, 804-807.

(35) Kim, H.; Kim, I.; Choi, H. J.; Kim, W. Thermal Conductivities of $\mathrm{Si}_{1 \mathrm{x}} \mathrm{Ge}_{\mathrm{x}}$ Nanowires with Different Germanium Concentrations and Diameters. Appl. Phys. Lett. 2010, 96, 2331061-2331063.
(36) Wang, Z.; Mingo, N. Diameter Dependence of SiGe Nanowire Thermal Conductivity. Appl. Phys. Lett. 2010, 97, 1019031-1019033.

(37) Lu, Q.; Adu, K. W.; Gutierrez, H. R.; Chen, G.; Lew, K. K.; Nimmatoori, P.; Zhang, X.; Dickey, E. C.; Redwing, J. M.; Eklund, P. C. Raman Scattering from $\mathrm{Si}_{1 \mathrm{x}} \mathrm{Ge}_{\mathrm{x}}$ Alloy Nanowires. J. Phys. Chem $\mathrm{C}$ 2008, 112, 3209-3215.

(38) Cahill, D. G.; Pohl, R. O. Thermal Conductivity of Amorphous Solids above the Plateau. Phys. Rev. B 1987, 35, 4067-4073.

(39) Dilhaire, S.; Pernot, G.; Calbris, G.; Rampnoux, J. M.; Grauby, S. Heterodyne Picosecond Thermoreflectance Applied to Nanoscale Thermal Metrology. J. Appl. Phys. 2011, 110, 114314.

(40) Tang, J.; Wang, H. T.; Lee, D. H.; Fardy, M.; Huo, Z.; Russell, T. P.; Yang, P. Holey Silicon as an Efficient Thermoelectric Material. Nano Lett. 2010, 10, 4279-4283.

(41) Doerk, G. S.; Carraro, C.; Maboudian, R. Single Nanowire Thermal Conductivity Measurements by Raman Thermography. ACS Nano 2010, 4, 4908-4914.

(42) Hippalgaonkar, K.; Huang, B.; Chen, R.; Sawyer, K.; Ercius, P.; Majumdar, A. Fabrication of Microdevices with Integrated Nanowires for Investigating Low Dimensional Phonon Transport. Nano Lett. 2010, 10, 4341-4348.

(43) Hopkins, P. E.; Rakich, P. T.; Olsson, R. H.; El kady, I. F.; Phinney, L. M. Origin of Reduction in Phonon Thermal Conductivity of Microporous Solids. Appl. Phys. Lett. 2009, 95, 1619021-1619023. 